\title{
RISK FACTORS FOR CORONARY ARTERY DISEASES: A STUDY AMONG PATIENTS WITH ISCHEMIC HEART DISEASE IN INDIA (KERALA)
}

\author{
Cyril James ${ }^{1,2}$, \\ Thankachan V. Attacheril', \\ N. Balakrishnan', \\ Diana K. Gaydarova², \\ Nadya Y. Stancheva ${ }^{2}$, \\ Ivan P. Gerchev ${ }^{2}$, \\ Shreya Ohri' ${ }^{2}$, \\ Snezhana T. Tisheva ${ }^{2}$ \\ ${ }^{1}$ Department of Cardiology, \\ Lourdes Heart Institute and Neuro \\ Center (LHINC), \\ Cochin, Kerala, \\ India \\ ${ }^{2}$ Department of Cardiology, \\ Medical University - Pleven, \\ Bulgaria
}

\begin{abstract}
Summary
Coronary artery disease (CAD) is a condition that develops due to accumulation of atherosclerotic plaque in the epicardial coronary arteries, leading to myocardial ischemia. It is the leading cause of death worldwide and is a common complex disease. A study was carried out in a group of 496 patients with acute coronary syndrome or with angiographic or stress test evidence for coronary artery disease, admitted to the Department of Cardiology at Lourdes Heart Institute and Neuro Centre during the period June -August 2012. The risk factors studied were hypertension, diabetes mellitus, dyslipidemia, body mass index, smoking and family history of CAD. The results demonstrated that in both males and females of the Indian population studies, diabetes and dyslipidemia were major risk factors for CAD, while hypertension was not a major risk factor. Therefore, early detection and treatment of diabetes mellitus and dyslipidemia play a vital role in prevention of CAD in Indian population.
\end{abstract}

Key words: coronary artery disease, risk factors, hypertension, diabetes mellitus, dyslipidemia, body mass index

\section{Introduction}

CAD is a condition resulting from accumulation of atherosclerotic plaque in the epicardial coronary arteries and leading to myocardial ischemia. It is a common multifarious public health issue today and a leading cause of morbidity and mortality in both developing and developed countries [1]. Cardiovascular disease affects millions of people in both developed and developing countries. Although the rate of death attributable to the disease has declined in developed countries in the past several decades, it is still the leading cause of death and extorts a heavy social and economic toll globally. In low and middle income countries, the prevalence of cardiovascular disease has increased dramatically. By 2020, the disease is forecasted to become the major cause of morbidity and mortality in most developing nations $[2,3]$. CAD includes a spectrum of disease manifestations ranging from

Received: November 14, 2012

Revision received: June 18, 2013

Accepted: June 26, 2013 
asymptomatic atherosclerotic disease to acute coronary syndrome, which includes ST elevation myocardial infarction (STEMI), Non-ST elevation myocardial infarction (NSTEMI) and unstable angina.

The risk factors for CAD are broadly classified as modifiable and non-modifiable risk factors. Modifiable risk factors include hypertension, diabetes mellitus, dyslipidemia, obesity and smoking. Non-modifiable risk factors include age, sex, race, and family history for CAD [4]. The Systematic Coronary Risk Evaluation system is recommended to assess an individual's total cardiovascular risk. CAD is closely related to lifestyle and modifiable physiological factors, and risk factor modification has been shown to reduce cardiovascular morbidity and mortality. CAD is the most common cause of mortality in India [5], homing approximately one-sixth of the world population. Hence understanding the predominant risk factors among the Indian population is important [6, 7]. Furthermore, the South Asian population, especially that of the Indian subcontinent, is believed to have a higher risk and prevalence of CAD as compared with European and African populations [8,9].

The prevalence and incidence of CAD along with the risk factor profile vary greatly across the regions of the world. Regional differences in the prevalence and incidence of atherosclerotic coronary disease may depend upon genetic variability, lifestyle differences and regional differences in medical care systems, etc.

A key factor that hampers the development of preventive strategies in developing countries such as India is the meagre amount $(8 \%)$ of published literature on CVD research available from these countries [10]. Much of the knowledge of risk factors for coronary artery disease (CAD) has been acquired from studies conducted on the Western population. It is widely believed that the association of these risk factors with CAD in other populations needs to be ascertained, and there is speculation that differences might range from the frequency of presence of classical risk factors to their total absence or irrelevance in these populations. Therefore, it is imperative to undertake large population-based, prospective studies in developing countries such as India to identify CAD risk factors, both conventional and novel. However, careful scrutiny of available scientific evidence for modifiable CAD risk factors (elevated serum total and low-density lipoprotein cholesterol [LDL-C], low high-density lipoprotein cholesterol [HDL-C], smoking, diabetes, hypertension, low level of physical activity, and obesity) in this population may be helpful in formulating a more relevant CAD prevention strategy. A cost-effective preventive strategy will need to focus on reducing risk factors both in the individual and in the population at large.

The aim of the study was to analyse the major risk factors among the Indian population in the State of Kerala for CAD.

\section{Patients and Methods}

The study was conducted at the Lourdes Heart Institute and Neuro Center (LHINC), a tertiary Hospital in Cochin in the state of Kerala located in the southernmost part of India. Ethical approval for the study was obtained from the Institutional Ethical Review Committee.

The study was carried out on 496 patients, admitted to the Department of Cardiology in the hospital during the study period from June 1, 2012 to August 31, 2012 and met the inclusion criteria. The inclusion criteria were:

- Acute coronary syndrome - STEMI, NSTEMI or unstable angina;

- Post-myocardial infarction state - with history of coronary bypass graft or percutaneous coronary intervention with or without stenting or with history of medical management either with fibrinolytics or with heparins;

- Chronic ischemic heart disease - evidence from coronary angiogram or from a positive stress test.

The data used for the study was collected from the patients and their previous medical records. Smoking was defined as use of bidis (small, thin hand-rolled cigarettes found primarily in India, consisting of tobacco, wrapped in leaves of tendu or temburni - plants native to Asia) or cigarettes. Physical examination of patients included height, weight, abdominal circumference and two blood pressure measurements: at the time of admission and on the following day. Hypertension was classified based on the JNC 7 Classification for Hypertension (reviewed) [11]. Laboratory investigations included random blood sugar, fasting blood sugar, 2-hour post prandial blood sugar, $\mathrm{HbA} 1 \mathrm{c}$, fasting lipid profile (total cholesterol, LDL cholesterol, HDL cholesterol and triglyceride level), and Troponin T and ECG. Patients were defined as diabetic or with impaired 
glucose tolerance based on their blood sugar levels and HbAlc values. Dyslipidemia was defined based on the Fasting Lipid Profile. Classification of the patients according to the inclusion criteria was made based on ECG findings, Troponin $T$ and medical records.

The acquired data were analyzed statistically using Microsoft Excel. In T-test analysis, P value $<0.05$ was considered as statistically significant.

\section{Results}

A total of 496 patients admitted to the Department of Cardiology in the hospital during the study period were analysed according to the study parameters. Of the total number of patients included in the study, 310 (62\%) were males and $186(38 \%)$ were females.

Acute coronary syndrome was established in $278(56 \%)$ patients, $184(31 \%)$ of the patients were with history of myocardial infarction and 64 $(13 \%)$ of the patients were with evidence of chronic ischemic heart disease (Figure 1).

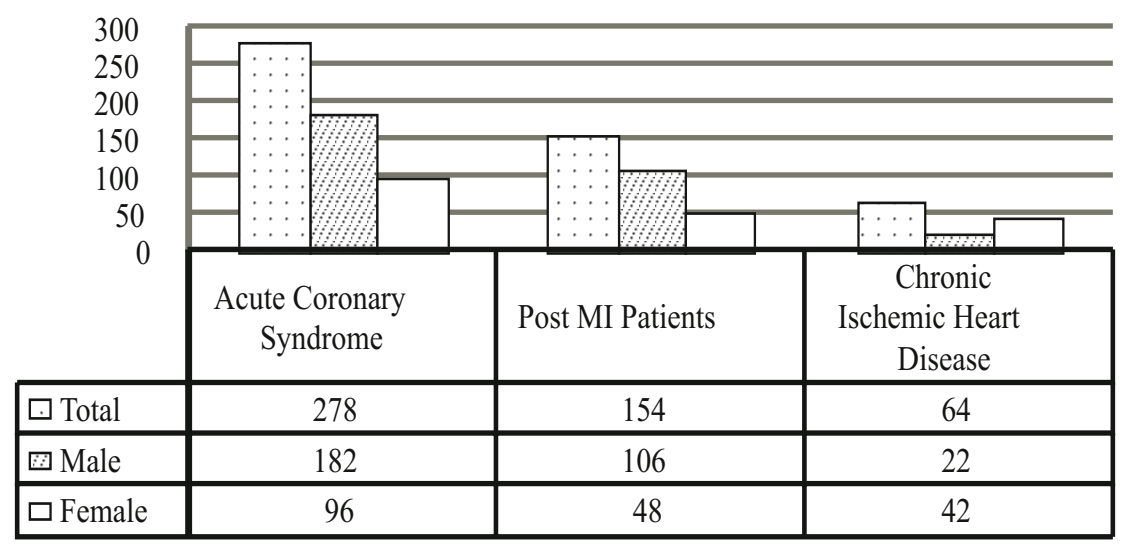

Figure 1. Distribution of study group by inclusion criteria and gender

Of the patients studied, $19(3.8 \%)$ were under the age of $35 ; 78(15.7 \%)$ were in the age group $35-44 ; 139(28 \%)$ were in the age group 45-54;

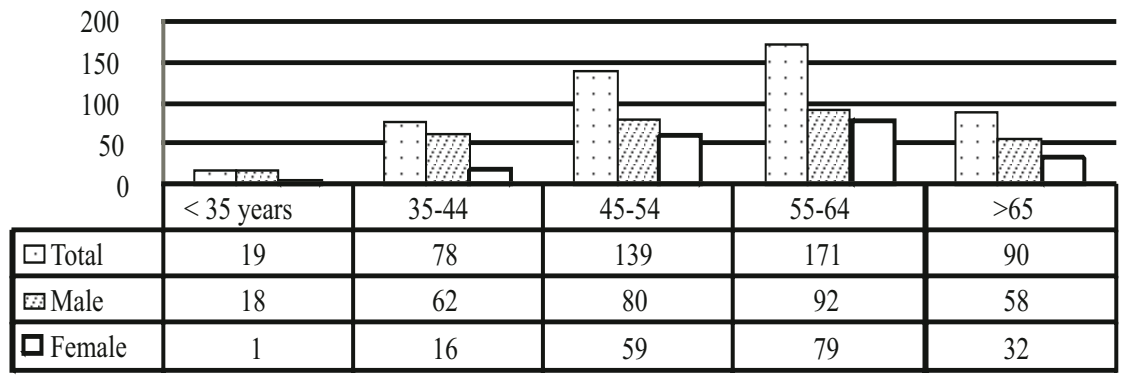

Figure 2. Distribution of study group based on age group

Peak incidence of CAD occurred between the age group 45-64, and the incidence of CAD was negligible in females below the age of $44(9 \%)$. The study showed that CAD in males started a decade earlier that in females. The incidence of CAD in males $(20 \%)$ was higher than in females $(8.6 \%)$ in the age group $35-45$. In patients older than 45 years, the male/female ratio of CAD
$171(34.4 \%)$ were in the age group 55-64, and 90 $(18.1 \%)$ were above the age of 65 (Figure 2 ). occurrence was the same (Figure 2).

A positive family history for CAD was found in $282(57 \%)$ patients in our study.

Based on blood pressure monitoring, the patients were classified according to JNC 7 (reviewed) Classification for Hypertension as normotensive 232 (47\%), pre-hypertensive $17(14 \%)$, stage I hypertensive $52(10 \%)$, stage II 
— James C. et al. Risk factors for coronary artery diseases: a study among patients with ischemic heart...

hypertensive $82(17 \%)$. Isolated systolic hypertension was found in 60 patients $(12 \%)$
(Figure 3) There was no gender difference noted in the occurrence of hypertension.

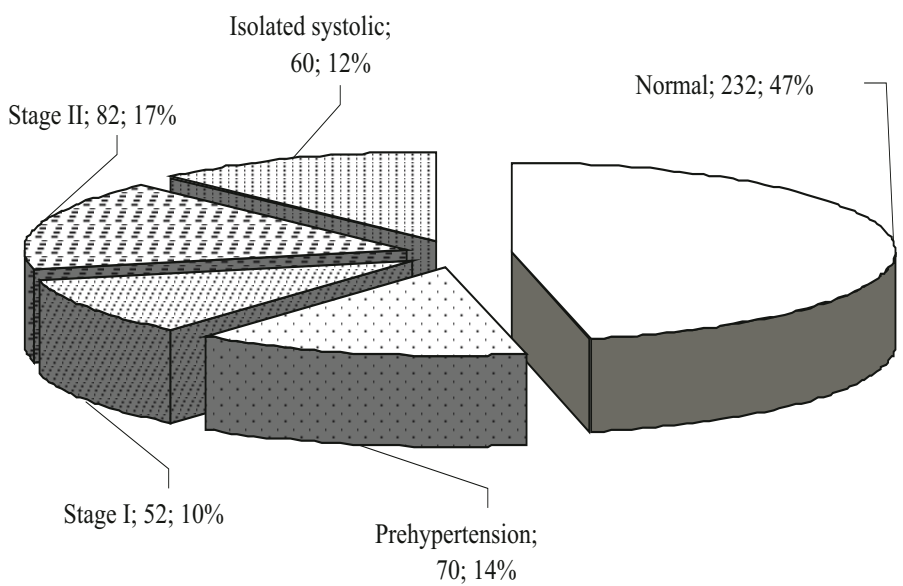

Figure 3. Distribution of patients based on blood pressure monitoring according to JNC 7 (reviewed) classification for hypertension

Diabetes mellitus was found to be a major risk factor in both males and females in the study population. Of all the 496 patients, $284(58 \%)$ had diabetes mellitus, and $102(21 \%)$ were with impaired glucose tolerance. Of the male patients, $166(54 \%)$ were diabetics, and $62(20 \%)$ were found with impaired glucose tolerance. For the female group, the values were $118(54 \%)$ and 40 $(20 \%)$, respectively (Figure 4 ).

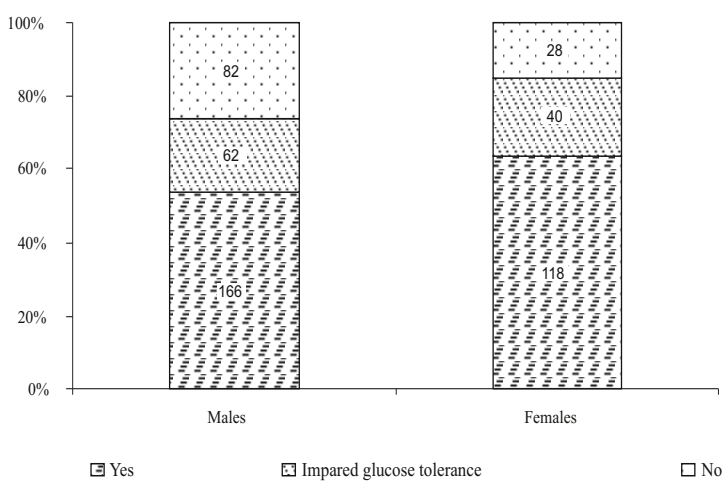

Figure 4. Diabetic status of the study population

Dyslipidemia was also a major risk factor along with diabetes mellitus for the Indian population. In the study group, the fasting lipid profile tests revealed evidence of dyslipidemia in $352(71 \%)$ of the patients: $206(66 \%)$ of the males and $146(78 \%)$ of the females. All the patients
$(100 \%)$ included in the study population reported using saturated oil for cooking, mainly coconut oil. This may be considered a causative factor for the high incidence of dyslipidemia among the study group (Figure 5).

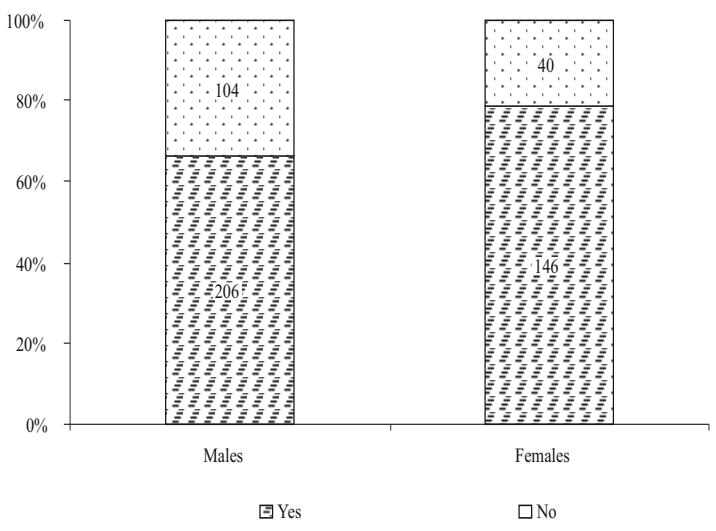

Figure 5. Dyslipidemia status of the study population

Of the study population, $378(76 \%)$ had never smoked in their lifetime, and only $40(8 \%)$ were current smokers. The number of female smokers in the study population was 0 .

Considering obesity as the risk factor for $\mathrm{CAD}$, based on the body mass index (BMI), only $50(16 \%)$ of the males had a BMI higher than 30 , whereas it was higher than 30 in $102(55 \%)$ of the females. Only $62(12 \%)$ of the studied population reported walking at least 30 minutes a day. 


\section{Discussion}

This study showed that CAD started in males a decade earlier than in females. The incidence of CAD in males $(20 \%)$ was higher as compared to that in females $(8.6 \%)$ in the age group $35-45$ $(\mathrm{P}<0.05)$. In the $45+$ age group, the male/female ratio for the occurrence of CAD was the same. In a study done by the World Health Organization, the male population was found to have approximately $40 \%$ increased CAD mortality as compared to the female population [12].

The peak incidence of CAD was seen in patients aged 45-64. Heart disease incidence increased in Asian Indians 5-10 years earlier than in other populations around the world. The mean age of first presentation of acute myocardial infarction in Indians is 53 years [13]. Coronary artery disease (CAD) that manifests at a younger age can have devastating consequences for an individual, family and society. A case-control study of risk factors for coronary heart disease in urban Indian middle-aged males, done by Jain et al. showed the presence of family history of premature CVD in $4.4 \%$ of patients [14].

A low incidence of hypertension (39\%) was seen among the study population. Hence hypertension was found to be an insignificant risk factor among the population studied. The prevalence of hypertension in India is low as compared to world figures. In India, $23.1 \%$ of men and $22.6 \%$ of women over 25 years old suffer from hypertension, says the WHO "Global health statistics 2012"[ 15]. Sofia Study and EUROSPIRE III have shown that with advancing age, all forms of CAD increase among Bulgarians. In Sofia Study and EUROSPIRE study hypertension has been seen as a major risk factor for CAD [16]. Vladimirov V. et al. found a high incidence $(56.3 \%)$ of hypertension among the Bulgarian population [17], in contrast to the low incidence of hypertension among the Indian population $(39 \%)$.

A high incidence of diabetes and impaired glucose tolerance were seen among the population we studied. Of the total 496 patients, 284 (58\%) had diabetes mellitus, and 102 (21\%) were with impaired glucose tolerance. Of the male patients, $166(54 \%)$ were diabetics, and 62 $(20 \%)$ were found with impaired glucose tolerance. In the female group, the values were $118(54 \%)$ and 40 (20\%), respectively. There was no statistically significant difference in the incidence of diabetes mellitus in the male and female patients with $\mathrm{CAD}(\mathrm{P}>0.05)$.

Indians are genetically prone to develop type II diabetes mellitus due to insulin resistance. The hyperinsulinimia in these patients accelerates the atherosclerotic process in the coronary arteries. Diabetes is second only to CAD as a health burden in India. During the past decade, the number of people with diabetes in India increased from 32 million to 50 million, and the projected figure may reach 87 million by 2030 [ 18]. Hyperinsulinaemia, insulin resistance, and the higher rate of prevalence of metabolic syndrome in people with type 2 diabetes were attributed to high coronary risk in south Asians [19, 20]. In Chennai (formerly Madras), India, an urban population study demonstrated that the prevalence rates for coronary artery disease were $9.1 \%$ in normal subjects and $21.4 \%$ in those with type 2 diabetes. According to the Interheart study, the risk for myocardial infarction attributable to diabetes was $9 \%$.

In the study group, the fasting lipid profile tests revealed dyslipidemia in $352(71 \%)$ of the patients: 206 (66\%) of the males and 146 (78\%) of the females. The study did not reveal a statistically significant difference in the incidence of dyslipidemia in male and female patients with CAD $(\mathrm{P}>0.05)$. The role of dyslipidemia in the pathogenesis of CAD is well known [21]. In a study conducted between 1998 and 2002 in a North Indian population, Goel et al. showed that CAD occured at much lower levels of total cholesterol and LDL-C than in other populations, and high triglyceride and low HDL levels were universal in this population. Our study revealed a high prevalence of dyslipidemia (71\%) - elevated levels of total cholesterol, LDL$\mathrm{C}$ and high triglycerides with concurrent low HDL-C values.

Positive family history for CAD was seen in $57 \%$ of the patients. A family history reflects both genetic susceptibility and interactions between genetic, environmental, cultural and behavioural factors [22]. Individuals with genetic susceptibility develop the disease at an earlier age. Early detection of CAD in these individuals may help to modify risk factors. Non-invasive methods, e.g. coronary calcium scoring, might help predicting CAD in these patients. Some studies indicate that positive family history is a predictor of impaired endothelium-dependent coronary blood flow regulation in humans [23].

Enas et al. have shown that Indian emigrants to Western states have a high prevalence of dyslipidemia and insulin resistance, thereby 
— James C. et al. Risk factors for coronary artery diseases: a study among patients with ischemic heart...

increasing the risk for CAD [24]. A modest increase in body fat with central distribution has

\section{References}

1. Lopez AD, Murray CC. The global burden of disease, 1990-2020. Nat Med. 1998;4(11):1241-3.

2. Reddy KS, Yusuf S. Emerging epidemic of cardiovascular disease in developing countries. Circulation. 1998;97(6):596-601.

3. Reddy KS. Cardiovascular disease in non-Western countries. N Engl J Med 2004;350(24):2438-40.

4. Yusuf S, Hawken S, Onupuu S, Dans T, Avezum A, Lanas F, et al. Effect of potentially modifiable risk factors associated with myocardial infarction in 52 countries (the INTERHEART study): case-control study. Lancet 2004;364(9438):937-52.

5. Gupta R. Burden of coronary heart disease in India. Indian Heart J. 2005;57(6):632-8.

6. Shah B, Mathur P. Surveillance of cardiovascular disease risk factors in India: the need \& scope. Indian J Med Res. 2010;132(5):634-42.

7. Ajay VS, Prabhakaran D. Coronary heart disease in Indians: implications of the INTERNET study. Indian J Med Res. 2010;132:561-6.

8. Goyal A, Yusuf S. The burden of cardiovascular disease in the Indian subcontinent. Indian J Med Res 2006;124(3):235-44.

9. Yusuf S, Reddy S, Ounpuu S, Anand S. Global

burden of cardiovascular diseases: Part II: variations in cardiovascular disease by specific ethnic groups and geographic regions and prevention strategies. Circulation. 2001;104(23):2855-64.

10. Mackay J, Mensah G, editors. The atlas of heart disease and stroke. Geneva: World Health Organization, Centers for Disease Control and Prevention; 2004.

11. Chobanian AV, Bakris GL, Black HR, Cushman WC, Green LA, Izzo JL Jr, et al. Seventh Report of the Joint National Committee on Prevention, Detection, Evaluation, and Treatment of High Blood Pressure. Hypertension. 2003;42(6):120652.

12. Jackson R, Chambles L, Higgins M, Kuulasmaa K, Wijnberg L, Williams D, WHO MONICA Project, and ARIC Study. Sex difference in ischaemic heart disease mortality and risk factors in 46 communities: an ecologic analysis. Cardiovasc Risk Fact. 1999; 7:43-54.

13. Enas EA, Garg A, Davidson MA, Nair VM, Huet BA, Yusuf S. Coronary heart disease and its risk factors in first-generation immigrant Asian Indians to the United States of America. Indian Heart J. 1996;48(4):343-53.

14. Jain P, Jain P, Bhandari S, Siddhu A. A case-control 
study of risk factors for coronary heart disease in urban Indian middle-aged males. Indian Heart J 2008;60(3):233-40.

15. WHO. World Health Statistics 2012. Global health statistics 2012. [cited 2013 Jan 27]. Available from: http://www.who.int/gho/publications/ world_health_statistics/2012/en

16. Jennings CS, Kotseva K, Amouyel P, Debacker G, Debacquer D, Keil U, et al. Reported lifestyle habits and lifestyle changes in high risk patients from 12 European regions: The EUROASPIRE III survey in general practice, Eur Heart J. 2009;30:508-9.

17. Vladimirov V, Draganov V, Krunchev N, Petkov P. [Risk factors in ischemic heart disease patients]. Vutr Boles. 1978;17(6):29-37. [In Bulgarian].

18. Sandeep S, Deepa R, Mohan V, Shah B, Varghese C. Epidemiology of type 2 diabetes: Indian scenario. Indian J Med Res. 2007;125(3):217-30.

19. Mohan V, Radhika G, Vijayalakshmi P, Sudha V. Can the diabetes/cardiovascular disease epidemic in India be explained, at least in part, by excess refined grain (rice) intake? Indian J Med Res. 2010;131:369-72.

20. McKeigue PM, Ferrie JE, Pierpoint T, Marmot MG. Association of early-onset coronary heart disease in South Asian men with glucose intolerance and hyper insulinemia. Circulation 1993;87(1):152-61.

21. Mohan V, Deepa R, Rani SS, Premalatha G; Chennai Urban Population Study (CUPS No.5). Prevalence of coronary artery disease and its relationship to lipids in a selected population in South India. J Am Coll Cardiol. 2001;38(3):682-7.

22. Scheuner MT. Genetic evaluation for coronary artery disease. Genet Med. 2003;5(4):269-85.
23. Schächinger V, Britten MB, Elsner M, Walter DH, Scharrer I, Zeiher AM. A positive family history of premature coronary artery disease is associated with impaired endothelium-dependent coronary blood flow regulation. Circulation 1999;100(14):1502-8.

24. Enas EA, Yusuf S, Sharma S. Coronary artery disease in South Asians: Second Meeting of the International Working Group 16 March 1997, Anaheim, California. Indian Heart J. 1998;50:105-11.

25. Gambhir JK, Kaur H, Prabhu KM, Morrisett JD, Gambhir DS. Association between lipoprotein(a) levels, apo(a) isoforms and family history of premature $\mathrm{CAD}$ in young Asian Indians. Clin Biochem. 2008;41(7-8):453-8.

26. Maitra A, Shanker J, Dash D, John S, Sannappa PR, Rao VS, et al. Polymorphisms in the IL6 gene in Asian Indian families with premature coronary artery disease - the Indian Atherosclerosis Research Study. Thromb Haemost. 2008;99(5):944-50.

27. Thompson PD, Buchner D, Pina IL, Balady GJ, Williams MA, Marcus BH, Berra K, et al. Exercise and physical activity in the prevention and treatment of atherosclerotic cardiovascular disease: a statement from the Council on Clinical Cardiology (Subcommittee on Exercise, Rehabilitation, and Prevention) and the Council on Nutrition, Physical Activity, and Metabolism (Subcommittee on Physical Activity). Circulation 2003;107(24):3109-16. 